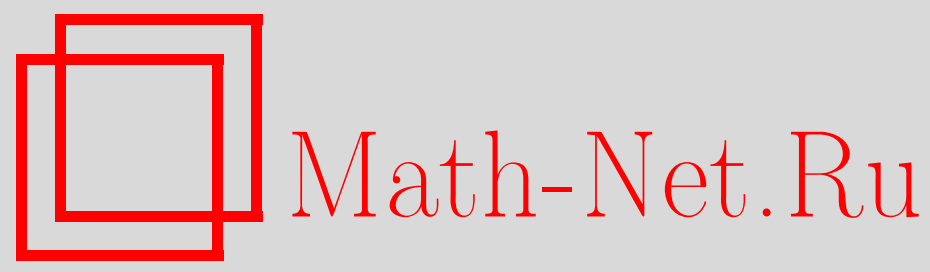

М. К. Потапов, О свойствах и о применении в теории приближений одного семейства операторов обобщенного сдвига, Матем. заметки, 2001, том 69, выпуск 3, 412-426

DOI: https://doi.org/10.4213/mzm514

Использование Общероссийского математического портала Math-Net.Ru подразумевает, что вы прочитали и согласны с пользовательским соглашением http://www.mathnet.ru/rus/agreement

Параметры загрузки:

IP: 54.92 .164 .108

26 апреля 2023 г., $17: 18: 09$

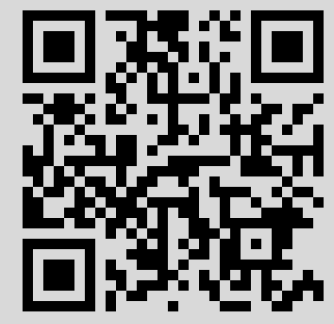




\section{О СВОЙСТВАХ И О ПРИМЕНЕНИИ В ТЕОРИИ ПРИБЛИЖЕНИЙ ОДНОГО СЕМЕЙСТВА ОПЕРАТОРОВ ОБОБЩЕННОГО СДВИГА}

\section{М.К. Потапов}

В работе определяется семейство новых несимметричных операторов обобщенного сдвига и приводятся их свойства. Для каждого из этих операторов вводится обобщенный модуль гладкости и для него приводятся прямая и обратная теоремы теории приближений.

Библиографоия: 13 названий.

Введение. Для $2 \pi$-периодических функций хорошо известны связи между ее обычным модулем гладкости и ее наилучшими приближениями тригонометрическими полиномами.

Для непериодических функций уже нет таких связей между ее обычным модулем гладкости и ее наилучшими приближениями алгебраическими многочленами. Однако полная аналогия с $2 \pi$-периодическим случаем имеет место тогда, когда обычньй модуль гладкости заменен обобщенным модулем гладкости.

Обобщенные модули гладкости могут определяться разными способами (см., например, [1]). Одним из таких способов является определение таких модулей при помощи операторов обобщенного сдвига [2]. Поскольку каждый оператор обобщенного сдвига действует в своем классе весовых пространств, актуальным является построение новых операторов обобщенного сдвига.

Ранее вводились и изучались симметричные операторы обобщенного сдвига (см., например, [3]-[9]).

В этой работе вводится одно семейство несимметричных операторов обобщенного сдвига, изучаются его свойства и показьвается его применение в теории приближений. В частном случае аналогичные результаты получены в [10].

Определения и обозначения. Обозначим через $L_{p}$, где $1 \leqslant p \leqslant \infty$, множество функций $f$, которые при $1 \leqslant p<\infty$ измеримы по Лебегу и суммируемы в $p$-й степени на отрезке $[-1,1]$, а при $p=\infty$ непрерьвны на отрезке $[-1,1]$, причем

$$
\|f\|_{p}= \begin{cases}\left(\int_{-1}^{1}|f(x)|^{p} d x\right)^{1 / p} & \text { при } 1 \leqslant p<\infty, \\ \max _{-1 \leqslant x \leqslant 1}|f(x)| & \text { при } p=\infty .\end{cases}
$$

Работа выполнена при поддержке Российского фонда фундаментальных исследований, грант № 99-01-00062, и программы поддержки ведуших научных школ, грант № 00-15-96143. 
Через $L_{p, \alpha, \beta}$ обозначим множество функций $f$ таких, что $f(x)(1-x)^{\alpha}(1+x)^{\beta} \in L_{p}$, причем

$$
\|f\|_{p, \alpha, \beta}=\left\|f(x)(1-x)^{\alpha}(1+x)^{\beta}\right\|_{p} .
$$

Через $E_{n}(f)_{p, \alpha, \beta}$ обозначим наилучшее приближение функции $f \in L_{p, \alpha, \beta}$ при помощи алгебраических многочленов $\mathscr{P}_{n-1}$ степени не выше, чем $n-1$, в метрике $L_{p, \alpha, \beta}$, т.е.

$$
E_{n}(f)_{p, \alpha, \beta}=\inf _{\mathscr{P}_{n-1}}\left\|f-\mathscr{P}_{n-1}\right\|_{p, \alpha, \beta}
$$

Для каждой пары натуральных чисел $s$ и $r$ введем оператор обобщенного сдвига

$$
T_{y}(f, x, r, s)=\frac{2^{s+r}}{\pi} \int_{-1}^{1} f(R) \psi(x, y, z) \frac{d z}{\sqrt{1-z^{2}}}
$$

где

$$
\begin{gathered}
\psi(x, y, z)=\frac{\cos \left[s\left(\varphi_{1}-\varphi\right)+r\left(\varphi_{1}-\mu\right)\right](1-R)^{r}\left(1-R^{2}\right)^{\frac{s}{2}}}{(1+y)^{s+r}(1-x)^{r}\left(1-x^{2}\right)^{\frac{s}{2}}}, \\
R=x y+z \sqrt{1-x^{2}} \sqrt{1-y^{2}} \\
\cos \varphi_{1}=z, \quad \sin \varphi_{1}=\sqrt{1-z^{2}} \\
\cos \varphi=\frac{-\sqrt{1-y^{2}} x+y z \sqrt{1-x^{2}}}{\sqrt{1-R^{2}}}, \quad \sin \varphi=\frac{\sqrt{1-x^{2}} \sqrt{1-z^{2}}}{\sqrt{1-R^{2}}} \\
\cos \mu=\frac{z(1-x y)-\sqrt{1-x^{2}} \sqrt{1-y^{2}}}{1-R}, \quad \sin \mu=\frac{\sqrt{1-z^{2}}(y-x)}{1-R} .
\end{gathered}
$$

Дальше будем считать фиксированными числа $s$ и $r$, т.е. считать фиксированным опеpaтор $T_{y}(f, x, r, s)$ и будем обозначать его через $T_{y}(f, x)$. При помощи этого оператора обобщенного сдвига для функции $f \in L_{p, \alpha, \beta}$ определим обобщенный модуль гладкости

$$
\widetilde{\omega}(f, \delta)_{p, \alpha, \beta}=\sup _{|t| \leqslant \delta}\left\|T_{\cos t}(f, x)-f(x)\right\|_{p, \alpha, \beta} .
$$

Обозначим через $\mathscr{D}_{u, \nu, \mu}$ оператор дифференцирования

$$
\mathscr{D}_{u, \nu, \mu}=\left(1-u^{2}\right) \frac{d^{2}}{d u^{2}}+(\mu-\nu-(\mu+\nu+2) u) \frac{d}{d u} .
$$

Будем писать, что $f \in \mathscr{A} \mathscr{D}(p, \alpha, \beta, \nu, \mu)$, если $f \in L_{p, \alpha, \beta}$ имеет абсолютно непрерьвную производную на каждом отрезке $[a, b] \subset(-1,1)$ и $\mathscr{D}_{x, \nu, \mu} f(x) \in L_{p, \alpha, \beta}$. Пусть

$$
K(f, \delta, \nu, \mu)_{p, \alpha, \beta}=\inf _{g \in \mathscr{A} \mathscr{D}(p, \alpha, \beta, \nu, \mu)}\left(\|f-g\|_{p, \alpha, \beta}+\delta^{2}\left\|\mathscr{D}_{x, \nu, \mu} g(x)\right\|_{p, \alpha, \beta}\right)
$$

есть $K$-функционал Петре, интерполируюший между пространствами $L_{p, \alpha, \beta}$ и $\mathscr{A} \mathscr{D}(p, \alpha, \beta, \nu, \mu)$.

Будем обозначать через $\mathscr{P}_{n}^{(\nu, \mu)}(x)(n=0,1,2, \ldots)$ многочлены Якоби, т.е. многочлены степени $n$, ортогональные друг другу с весом $(1-x)^{\nu}(1+x)^{\mu}$ на отрезке $[-1,1]$ и нормированные условием $\mathscr{P}_{n}^{(\nu, \mu)}(1)=1$.

Через $a_{n}(f)$ обозначим коэффициенты Фурье-Якоби функции $f \in L_{1, s+2 r, s}$ по системе многочленов Якоби $\left\{\mathscr{P}_{n}^{(s+2 r, s)}(x)\right\}_{n=0}^{\infty}$, т.е.

$$
a_{n}(f)=\int_{-1}^{1} f(x) \mathscr{P}_{n}^{(s+2 r, s)}(x)(1-x)^{s+2 r}(1+x)^{s} d x, \quad n=0,1,2, \ldots
$$




\section{Свойства оператора.}

Теорема 1. Oператор $T_{y}(f, x)$ обладает следующими свойствами:

1) оператор $T_{y}(f, x)$ линеен по $f$;

2) $T_{1}(f, x)=f(x)$;

3) $T_{y}\left(\mathscr{P}_{n}^{(s+2 r, s)}, x\right)=\mathscr{P}_{n}^{(s+2 r, s)}(x) \cdot \mathscr{P}_{n}^{(0,2 s+2 r)}(y), n=0,1,2, \ldots$;

4) $T_{y}(1, x)=1$;

5) если $g(x) T_{y}(f, x) \in L_{1, s+2 r, s}$ и $f(x) T_{y}(g, x) \in L_{1, s+2 r, s}$ для любого $y \in(-1,1)$, mo

$$
\begin{array}{rl}
\int_{-1}^{1} & f(x) T_{y}(g, x)(1-x)^{s+2 r}(1+x)^{s} d x \\
\quad=\int_{-1}^{1} g(x) T_{y}(f, x)(1-x)^{s+2 r}(1+x)^{s} d x
\end{array}
$$

6) $a_{n}\left(T_{y}(f, x)\right)=a_{n}(f) \cdot \mathscr{P}_{n}^{(0,2 s+2 r)}(y), n=0,1,2, \ldots$.

ДокАЗАТЕЛЬСтво. Свойства 1) и 2) следуют сразу из определения оператора $T_{y}(x)$. Для доказательства свойства 3) рассмотрим функции (см. [11, с. 133])

$$
P_{\lambda, \nu}^{l}(z)=\mathscr{P}_{n}^{(\alpha, \beta)}(z) 2^{-\lambda}\left(\begin{array}{c}
n+\alpha \\
n
\end{array}\right) i^{\lambda-\nu} \sqrt{\frac{(l-\lambda) !(l+\lambda) !}{(l-\nu) !(l+\nu) !}}(1-z)^{(\lambda-\nu) / 2}(1+z)^{(\lambda+\nu) / 2}
$$

где $l=n+(\alpha+\beta) / 2, \lambda=(\alpha+\beta) / 2, \nu=(\beta-\alpha) / 2$.

Для этих функций справедлива формула умножения (см. [11, с. 140]):

$$
P_{m, q}^{l}\left(\cos \theta_{1}\right) P_{q, r}^{l}\left(\cos \theta_{2}\right)=\frac{1}{2 \pi} \int_{-\pi}^{\pi} e^{i\left(q \varphi_{2}-(m-r) \varphi-r(\varphi+\eta)\right)} P_{m, r}^{l}(\cos \theta) d \varphi_{2}
$$

где

$$
\begin{gathered}
\cos \theta=\cos \theta_{1} \cos \theta_{2}-\sin \theta_{1} \sin \theta_{2} \cos \varphi_{2}, \\
e^{i \varphi}=\frac{\sin \theta_{1} \cos \theta_{2}+\cos \theta_{1} \sin \theta_{2} \cos \varphi_{2}+i \sin \theta_{2} \sin \varphi_{2}}{\sin \theta} \\
e^{i(\varphi+\eta)}=\frac{-\sin \theta_{1} \sin \theta_{2}+\left(1+\cos \theta_{1} \cos \theta_{2}\right) \cos \varphi_{2}+i\left(\cos \theta_{1}+\cos \theta_{2}\right) \sin \varphi_{2}}{1+\cos \theta} .
\end{gathered}
$$

Так как можно считать, что $\varphi=\varphi\left(\varphi_{2}\right), \mu=\varphi+\eta=\mu\left(\varphi_{2}\right), \theta=\theta\left(\varphi_{2}\right)$, легко видеть, что $\varphi\left(-\varphi_{2}\right)=-\varphi\left(\varphi_{2}\right), \mu\left(-\varphi_{2}\right)=-\mu\left(\varphi_{2}\right), \theta\left(-\varphi_{2}\right)=\theta\left(\varphi_{2}\right)$. Поэтому

$$
P_{m, q}^{l}\left(\cos \theta_{1}\right) P_{q, r}^{l}\left(\cos \theta_{2}\right)=\frac{1}{\pi} \int_{0}^{\pi} \cos \left(q \varphi_{2}-(m-r) \varphi-r(\varphi+\eta)\right) P_{m, r}^{l}(\cos \theta) d \varphi_{2}
$$

Полагая в этой формуле $m=q=s+r, \mu=\varphi+\eta$ и применяя формулу (2), имеем

$$
\mathscr{P}_{n}^{(s, s+2 r)}\left(\cos \theta_{2}\right) \mathscr{P}_{n}^{(0,2 s+2 r)}\left(\cos \theta_{1}\right)=\frac{2^{s+r}}{\pi} \int_{0}^{\pi} \mathscr{P}_{n}^{(s, s+2 r)}(\cos \theta) \psi_{1} d \varphi_{2},
$$


где

$$
\psi_{1}=\frac{(\sin \theta)^{s}(1+\cos \theta)^{r} \cos \left(s\left(\varphi_{1}-\varphi\right)+r\left(\varphi_{1}-\mu\right)\right)}{\left(1+\cos \theta_{1}\right)^{s+r}\left(\sin \theta_{2}\right)^{s}\left(1+\cos \theta_{2}\right)^{r}} .
$$

Сделав сначала замену переменных $\theta_{2}=\pi-\theta_{3}$, учитывая (см. [12, с. 171]), что

$$
\mathscr{P}_{n}^{(s, s+2 r)}(-z)=(-1)^{n} \mathscr{P}_{n}^{(s+2 r, s)}(z),
$$

и обозначая $\cos \theta_{3}=x, \cos \theta_{1}=y, \cos \varphi_{2}=z$, получаем, что

$$
\mathscr{P}_{n}^{(s+2 r, s)}(x) \mathscr{P}_{n}^{(0,2 s+2 r)}(y)=\frac{2^{s+r}}{\pi} \int_{-1}^{1} \mathscr{P}_{n}^{(s+2 r, s)}(R) \psi(x, y, z) \frac{d z}{\sqrt{1-z^{2}}}
$$

где $\psi(x, y, z), R, \varphi$ и $\mu$ определены формулами (1). Равенство (3) означает справедливость свойства 3 ).

Справедливость свойства 4) вытекает из справедливости свойства 3) при $n=0$.

Докажем теперь свойство 5). Рассмотрим

$$
\begin{aligned}
I & =\int_{-1}^{1} f(x) T_{y}(g, x)(1-x)^{s+2 r}(1+x)^{s} d x \\
& =\frac{2^{s+r}}{\pi} \int_{-1}^{1} \int_{-1}^{1} f(x) g(R) \psi(x, y, z)(1-x)^{s+2 r}(1+x)^{s} \frac{d z d x}{\sqrt{1-z^{2}}}
\end{aligned}
$$

где $\psi, R, \mu, \varphi$ и $\varphi_{1}$ определены формулами (1). Поскольку $\mu, \varphi$ и $\varphi_{1}$ являются функциями от $x, y$ и $z$, то, обозначая $\lambda=\lambda(x, y, z)=\varphi-\varphi_{1}, \chi=\chi(x, y, z)=\mu-\varphi_{1}$, получим, что

$$
\psi(x, y, z)=\frac{\cos (s \lambda+r \chi)(1-R)^{r}\left(1-R^{2}\right)^{s / 2}}{(1+y)^{s+r}(1-x)^{r}\left(1-x^{2}\right)^{s / 2}}
$$

Сделав в двойном интеграле замену переменных

$$
x=R y+\nu \sqrt{1-R^{2}} \sqrt{1-y^{2}}, \quad z=\frac{R \sqrt{1-y^{2}}-\nu y \sqrt{1-R^{2}}}{\sqrt{1-\left(R y+\nu \sqrt{1-R^{2}} \sqrt{1-y^{2}}\right)^{2}}}
$$

получим, что

$$
I=\int_{-1}^{1} g(R)\left(\frac{2^{s+r}}{\pi} \int_{-1}^{1} f(x) \psi_{1}(R, y, \nu) \frac{d \nu}{\sqrt{1-\nu^{2}}}\right)(1-R)^{s+2 r}(1+R)^{s} d R
$$

где

$$
\psi_{1}(R, y, \nu)=\frac{\cos (s \bar{\lambda}+r \bar{\chi})(1-x)^{r}\left(1-x^{2}\right)^{s / 2}}{(1+y)^{s+r}(1-R)^{r}\left(1-R^{2}\right)^{s / 2}}
$$

$\bar{\lambda}$ и $\bar{\chi}-$ функции от $R, y, \nu$.

Поскольку можно показать, что $\cos \bar{\lambda}=\cos \lambda(R, y, \nu), \sin \bar{\lambda}=\sin \lambda(R, y, \nu), \cos \bar{\chi}=$ $\cos \chi(R, y, \nu), \sin \bar{\chi}=\sin \chi(R, y, \nu)$, справедливо равенство

$$
\cos (s \bar{\lambda}+r \bar{\chi})=\cos (s \lambda(R, y, \nu)+r \chi(R, y, \nu))
$$


Это равенство означает, что $\psi_{1}(R, y, \nu)=\psi(R, y, \nu)$, и поэтому из $(5)$ получаем, что

$$
I=\int_{-1}^{1} g(R) T_{y}(f, R)(1-R)^{s+2 r}(1+R)^{s} d R
$$

т.е. получаем справедливость свойства 5).

Для доказательства свойства 6) рассмотрим

$$
I_{1}=a_{n}\left(T_{y}(f, x)\right)=\int_{-1}^{1} T_{y}(f, x) \mathscr{P}_{n}^{(s+2 r, s)}(x)(1-x)^{s+2 r}(1+x)^{s} d x .
$$

Используя доказанные вьше свойства 5) и 3), получаем, что

$$
\begin{aligned}
I_{1} & =\int_{-1}^{1} f(x) T_{y}\left(\mathscr{P}_{n}^{(s+2 r, s)}, x\right)(1-x)^{s+2 r}(1+x)^{s} d x \\
& =\mathscr{P}_{n}^{(0,2 s+2 r)}(y) \int_{-1}^{1} f(x) \mathscr{P}_{n}^{(s+2 r, s)}(x)(1-x)^{s+2 r}(1+x)^{s} d x=\mathscr{P}_{n}^{(0,2 s+2 r)}(y) a_{n}(f)
\end{aligned}
$$

и свойство 6) доказано.

ТЕОРема 2. Пусть даны числа $s, r, p$ и а такие, что s ur-натуральные числа, $1 \leqslant p \leqslant \infty, s / 2-1 / 2<\alpha \leqslant s / 2$ npu $p=1, s / 2-1 /(2 p)<\alpha<s / 2+1 / 2-1 /(2 p) n p u$ $1<p<\infty, s / 2 \leqslant \alpha<s / 2+1 / 2$ при $p=\infty$. Пусть функиия $f \in L_{p, \alpha+r, \alpha}$. Тогда справедливо неравенство

$$
\left\|T_{y}(f, x)\right\|_{p, \alpha+r, \alpha} \leqslant \frac{C}{(1+y)^{s+r}}\|f\|_{p, \alpha+r, \alpha}
$$

где постоянная $C$ не зависит от $f$ и $y \in(-1,1)$.

ДокАЗАТЕЛЬСтво. Пусть $p=1$. Рассмотрим

$$
J=\left\|T_{y}(f, x)\right\|_{1, \alpha+r, \alpha} \leqslant \frac{2^{s+r}}{\pi} \int_{-1}^{1} \int_{-1}^{1}|f(R)||\psi(x, y, z)|(1-x)^{\alpha+r}(1+x)^{\alpha} \frac{d z d x}{\sqrt{1-z^{2}}}
$$

где функция $\psi$ определена формулами (2). Так как

$$
|\psi(x, y, z)| \leqslant \frac{(1-R)^{r}\left(1-R^{2}\right)^{s / 2}}{(1+y)^{s+r}(1-x)^{r}\left(1-x^{2}\right)^{s / 2}}
$$

TO

$$
\begin{aligned}
J \leqslant & \frac{2^{s+r}}{\pi(1+y)^{s+r}} \\
& \times \int_{-1}^{1} \int_{-1}^{1}|f(R)|\left(1-R^{2}\right)^{s / 2}(1-R)^{r}\left(\left(1-x^{2}\right)\left(1-z^{2}\right)\right)^{\alpha-s / 2}\left(1-z^{2}\right)^{s / 2-\alpha} \frac{d z d x}{\sqrt{1-z^{2}}} .
\end{aligned}
$$

Поскольку $\alpha \leqslant s / 2$, то $\left(1-z^{2}\right)^{s / 2-\alpha} \leqslant 1$. Поэтому

$$
J \leqslant \frac{2^{s+r}}{\pi(1+y)^{s+r}} \int_{-1}^{1} \int_{-1}^{1}|f(R)|\left(1-R^{2}\right)^{s / 2}(1-R)^{r}\left(\left(1-x^{2}\right)\left(1-z^{2}\right)\right)^{\alpha-s / 2} \frac{d z d x}{\sqrt{1-z^{2}}} .
$$


Сделав в двойном интеграле замену переменных по формулам (4), получим

$$
\begin{aligned}
J \leqslant & \frac{2^{s+r}}{\pi(1+y)^{s+r}} \\
& \times \int_{-1}^{1} \int_{-1}^{1}|f(R)|\left(1-R^{2}\right)^{s / 2}(1-R)^{r}\left(\left(1-R^{2}\right)\left(1-\nu^{2}\right)\right)^{\alpha-s / 2} \frac{d R d \nu}{\sqrt{1-\nu^{2}}} \\
= & \frac{2^{s+r}}{\pi(1+y)^{s+r}} \int_{-1}^{1}|f(R)|(1-R)^{\alpha+r}(1+R)^{\alpha} d R \int_{-1}^{1}\left(1-\nu^{2}\right)^{\alpha-s / 2-1 / 2} d \nu
\end{aligned}
$$

Так как $\alpha>s / 2-1 / 2$, то

$$
\int_{-1}^{1}\left(1-\nu^{2}\right)^{\alpha-s / 2-1 / 2} d \nu=C_{1}
$$

Поэтому

$$
J \leqslant \frac{C_{2}}{(1+y)^{s+r}} \int_{-1}^{1}|f(R)|(1-R)^{\alpha+r}(1+R)^{\alpha} d R=\frac{C_{2}}{(1+y)^{s+r}}\|f\|_{1, \alpha+r, \alpha}
$$

и теорема 2 доказана для $p=1$.

Пусть $1<p<\infty$. Рассмотрим

$$
\begin{aligned}
J^{p} & =\left\|T_{y}(f, x)\right\|_{p, \alpha+r, \alpha}^{p} \\
& \leqslant\left(\frac{2^{s+r}}{\pi}\right)^{p} \int_{-1}^{1}\left(\int_{-1}^{1}|f(R)||\psi(x, y, z)|(1-x)^{\alpha+r}(1+x)^{\alpha} \frac{d z}{\sqrt{1-z^{2}}}\right)^{p} d x .
\end{aligned}
$$

Применяя оценку $(6)$, имеем

$$
\begin{aligned}
J^{p} \leqslant & \frac{2^{(s+r) p}}{\pi^{p}(1+y)^{(s+r) p}} \int_{-1}^{1}\left(\int_{-1}^{1}|f(R)|(1-R)^{r}\left(1-R^{2}\right)^{s / 2}\left(\left(1-x^{2}\right)\left(1-z^{2}\right)\right)^{\alpha-s / 2}\right. \\
& \left.\times\left(1-z^{2}\right)^{1 /(2 p)-\alpha+s / 2-1 / 2} \frac{d z}{\left(1-z^{2}\right)^{1 /(2 p)}}\right)^{p} d x .
\end{aligned}
$$

Применяя неравенство Гёльдера к внутреннему интегралу, получаем

$$
\begin{aligned}
J^{p} & \leqslant \frac{2^{(s+r) p}}{\pi^{p}(1+y)^{(s+r) p}} \int_{-1}^{1} \int_{-1}^{1}\left(|f(R)|(1-R)^{r}\left(1-R^{2}\right)^{s / 2}\right. \\
& \left.\times\left(\left(1-x^{2}\right)\left(1-z^{2}\right)\right)^{\alpha-s / 2}\right)^{p} \frac{d z d x}{\sqrt{1-z^{2}}}\left(\int_{-1}^{1}\left(1-z^{2}\right)^{(1 /(2 p)-\alpha+s / 2-1 / 2) p /(p-1)} d z\right)^{p-1} .
\end{aligned}
$$

Так как $\alpha<s / 2+1 / 2-1 /(2 p)$, то

$$
\left(\int_{-1}^{1}\left(1-z^{2}\right)^{(1 /(2 p)-\alpha+s / 2-1 / 2) p /(p-1)} d z\right)^{p-1}=C_{3}
$$


Поэтому

$$
\begin{aligned}
J^{p} \leqslant & \frac{C_{4}}{(1+y)^{(s+r) p}} \\
& \times \int_{-1}^{1} \int_{-1}^{1}\left(|f(R)|(1-R)^{r}\left(1-R^{2}\right)^{s / 2}\left(\left(1-x^{2}\right)\left(1-z^{2}\right)\right)^{\alpha-s / 2}\right)^{p} \frac{d z d x}{\left(1-z^{2}\right)^{1 / 2}} .
\end{aligned}
$$

Сделав в двойном интеграле замену переменных по формулам (4), имеем

$$
J^{p} \leqslant \frac{C_{4}}{(1+y)^{(s+r) p}} \int_{-1}^{1}\left(|f(R)|(1-R)^{\alpha+r}(1+R)^{\alpha}\right)^{p} d R \int_{-1}^{1}\left(1-\nu^{2}\right)^{(\alpha-s / 2) p-1 / 2} d \nu .
$$

Поскольку $\alpha>s / 2-1 /(2 p)$, то

$$
\int_{-1}^{1}\left(1-\nu^{2}\right)^{(\alpha-s / 2) p-1 / 2} d \nu=C_{5}
$$

Поэтому

откуда следует, что

$$
J^{p} \leqslant \frac{C_{6}}{(1+y)^{(s+r) p}} \int_{-1}^{1}\left(|f(R)|(1-R)^{\alpha+r}(1+R)^{\alpha}\right)^{p} d R
$$

$$
J \leqslant \frac{C_{7}}{(1+y)^{s+r}}\|f\|_{p, \alpha+r, \alpha}
$$

и теорема 2 доказана для $p \in(1, \infty)$.

Пусть $p=\infty$. Рассмотрим

$$
I_{1}=\frac{2^{s+r}}{\pi}\left|\int_{-1}^{1} f(R) \psi(x, y, z) \frac{d z}{\sqrt{1-z^{2}}}\right|(1-x)^{\alpha+r}(1+x)^{\alpha} .
$$

Учитьвая оценку (6), имеем

$$
I_{1} \leqslant \frac{2^{s+r}}{\pi(1+y)^{s+r}} \int_{-1}^{1}|f(R)|(1-R)^{r}\left(1-R^{2}\right)^{\frac{s}{2}}\left(1-x^{2}\right)^{\alpha-\frac{s}{2}} \frac{d z}{\sqrt{1-z^{2}}} .
$$

Легко проверить, что

$$
\left(1-x^{2}\right)\left(1-z^{2}\right)=1-R^{2}-\left(x \sqrt{1-y^{2}}-z y \sqrt{1-x^{2}}\right)^{2} .
$$

Откуда $\left(1-x^{2}\right) \leqslant\left(1-R^{2}\right) /\left(1-z^{2}\right)$. Применяя эту оценку и учитьвая, что $\alpha \geqslant s / 2$, имеем

$$
\begin{aligned}
I_{1} & \leqslant \frac{2^{s+r}}{\pi(1+y)^{s+r}} \int_{-1}^{1}|f(R)|(1-R)^{\alpha+r}(1+R)^{\alpha}\left(1-z^{2}\right)^{-\alpha+s / 2-1 / 2} d z \\
& \leqslant \frac{2^{s+r}}{\pi(1+y)^{s+r}}\|f\|_{\infty, \alpha+r, \alpha} \int_{-1}^{1}\left(1-z^{2}\right)^{-\alpha+s / 2-1 / 2} d z .
\end{aligned}
$$

Поскольку $\alpha<s / 2+1 / 2$, то $\int_{-1}^{1}\left(1-z^{2}\right)^{-\alpha+s / 2-1 / 2} d z=C_{8}$. Поэтому

$$
I_{1} \leqslant \frac{C_{9}}{(1+y)^{s+r}}\|f\|_{\infty, \alpha+r, \alpha} .
$$

Так как $J=\left\|T_{y}(f, x)\right\|_{\infty, \alpha+r, \alpha}=\max _{-1 \leqslant x \leqslant 1} I_{1}$, отсюда следует, что

$$
J \leqslant \frac{C_{9}}{(1+y)^{s+r}}\|f\|_{\infty, \alpha+r, \alpha},
$$

и теорема 2 доказана для $p=\infty$. 


\section{Оценки модуля гладкости через $K$-функционал.}

Лемма 1. Пусть функиия $f(x)$ имеет на каждом отрезке $[a, b] \subset(-1,1)$ абсолютно непрерывную производную $f^{\prime}(x)$ и $D_{x, s+2 r, s} f(x) \in L_{1, s+2 r, s}$. Тогда при любом $y \in(-1,1)$ для почти всех $x \in(-1,1)$ справедливы равенства:

1) $T_{y}\left(D_{x, s+2 r, s} f(x), x\right)=D_{x, s+2 r, s}\left(T_{y}(f, x)\right)$,

2) $T_{y}(f, x)-f(x)=\int_{1}^{y}(1-v)^{-1}(1+v)^{-(2 s+2 r+1)}$

$$
\times \int_{1}^{v}(1+u)^{2 s+2 r} T_{u}\left(D_{x, s+2 r, s} f(x), x\right) d u d v
$$

3) $T_{y}(f, x)-T_{0}(f, x)=-\int_{1}^{y}(1-v)^{-1}(1+v)^{-(2 s+2 r+1)}$

$$
\times \int_{v}^{-1}(1+u)^{2 s+2 r} T_{u}\left(D_{x, s+2 r, s} f(x), x\right) d u d v .
$$

ДокАЗАТЕЛЬСТво леммы 1 проводится стандартными рассуждениями (см., например, [10]) и поэтому опускается.

СледСтвиЕ. Пусть функиия $f(x)$ имеет на каждом отрезке $[a, b] \subset(-1,1)$ абсолютно непрерывную производную $f^{\prime}(x)$ и $D_{x, s+2 r, s} f(x) \in L_{1, s+2 r, s}$. Тогда при любом $t \in(-\pi, \pi)$ для почти всех $x \in(-1,1)$ справедливы равенства

$$
\begin{aligned}
T_{\cos t}(f, x)-f(x)= & \int_{0}^{t}\left(\sin \frac{v}{2}\right)^{-1}\left(\cos \frac{v}{2}\right)^{-(4 s+4 r+1)} \\
& \times \int_{0}^{v} T_{\cos u}\left(D_{x, s+2 r, s} f(x), x\right) \sin \frac{u}{2}\left(\cos \frac{u}{2}\right)^{4 s+4 r+1} d u d v
\end{aligned}
$$

$u$

$$
\begin{aligned}
T_{\cos t}(f, x)-T_{0}(f, x)=- & \int_{\pi / 2}^{t}\left(\sin \frac{v}{2}\right)^{-1}\left(\cos \frac{v}{2}\right)^{-(4 s+4 r+1)} \\
& \times \int_{v}^{\pi} T_{\cos u}\left(D_{x, s+2 r, s} f(x), x\right) \sin \frac{u}{2}\left(\cos \frac{u}{2}\right)^{4 s+4 r+1} d u d v .
\end{aligned}
$$

ДокАЗАТЕЛЬСтво. Первое равенство следует из второго равенства леммы 1 подстановкой $\cos u$ и $\cos v$ вместо $u$ и $v$ соответственно. Аналогично второе равенство следует из третьего равенства леммы 1.

Tеорема 3. Пусть даны числа $s, r, p$ u $\alpha$ такие, что s u $r$ - натуральные числа, $1 \leqslant p \leqslant \infty, s / 2-1 / 2<\alpha \leqslant s / 2$ npu $p=1, s / 2-1 /(2 p)<\alpha<s / 2-1 /(2 p)+1 / 2$ npu $1<p<\infty, s / 2 \leqslant \alpha<s / 2+1 / 2$ при $p=\infty$. Пусть $f \in L_{p, \alpha+r, \alpha}$. Тогда для любого $\delta \in(0, \pi)$ справедливы неравенства

$$
C_{1} K(f, \delta, s+2 r, s)_{p, \alpha+r, \alpha} \leqslant \widetilde{\omega}(f, \delta)_{p, \alpha+r, \alpha} \leqslant \frac{C_{2}}{\left(\cos \frac{\delta}{2}\right)^{2 s+2 r}} K(f, \delta, s+2 r, s)_{p, \alpha+r, \alpha},
$$

әде положительные постоянные $C_{1} u C_{2}$ не зависят от $\delta u f$. 
ДокАЗАТЕЛЬСТво. Покажем, что для любой функции $g \in A D(p, \alpha+r, \alpha, s+2 r, s)$ и любого $t \in(-\pi, \pi)$ справедливо неравенство

$$
\left\|T_{\cos t}(g, x)-g(x)\right\|_{p, \alpha+r, \alpha} \leqslant \frac{C_{3}}{\left(\cos \frac{t}{2}\right)^{2 s+2 r}} t^{2}\left\|D_{x, s+2 r, s} g(x)\right\|_{p, \alpha+r, \alpha}
$$

где постоянная $C_{3}$ не зависит от $t$ и $g$.

Пусть $0<t \leqslant \pi / 2$. Тогда по следствию из леммы 1 , применяя обобщенное неравенство Минковского и теорему 2 , получим

$$
\begin{aligned}
I_{1}= & \left\|T_{\cos t}(g, x)-g(x)\right\|_{p, \alpha+r, \alpha} \\
\leqslant & \int_{0}^{t}\left(\sin \frac{v}{2}\right)^{-1}\left(\cos \frac{v}{2}\right)^{-(4 s+4 r+1)} \\
& \times \int_{0}^{v}\left\|T_{\cos u}\left(D_{x, s+2 r, s} f(x), x\right)\right\|_{p, \alpha+r, \alpha} \sin \frac{u}{2}\left(\cos \frac{u}{2}\right)^{4 s+4 r+1} d u d v \\
\leqslant & C_{4}\left\|D_{x, s+2 r, s} f(x)\right\|_{p, \alpha+r, \alpha} \\
& \times \int_{0}^{t}\left(\sin \frac{v}{2}\right)^{-1}\left(\cos \frac{v}{2}\right)^{-(4 s+4 r+1)} \int_{0}^{v} \sin \frac{u}{2}\left(\cos \frac{u}{2}\right)^{2 s+2 r+1} d u d v .
\end{aligned}
$$

Так как для $0<t \leqslant \pi / 2$ имеем

$$
\int_{0}^{t}\left(\sin \frac{v}{2}\right)^{-1}\left(\cos \frac{v}{2}\right)^{-(4 s+4 r+1)} \int_{0}^{v} \sin \frac{u}{2}\left(\cos \frac{u}{2}\right)^{2 s+2 r+1} d u d v \leqslant \frac{C_{5} t^{2}}{\left(\cos \frac{t}{2}\right)^{2 s+2 r}}
$$

то, учитьвая это неравенство, получаем, что

$$
I_{1} \leqslant \frac{C_{6} t^{2}}{\left(\cos \frac{t}{2}\right)^{2 s+2 r}}\left\|D_{x, s+2 r, s} f(x)\right\|_{p, \alpha+r, \alpha} .
$$

Пусть $\pi / 2 \leqslant t<\pi$. Тогда по следствию из леммы 1 , применяя обобщенное неравенство Минковского и теорему 2 , получим, что

$$
\begin{aligned}
I_{2}= & \left\|T_{\cos t}(g, x)-T_{0}(g, x)\right\|_{p, \alpha+r, \alpha} \\
\leqslant & C_{7}\left\|D_{x, s+2 r, s} f(x)\right\|_{p, \alpha+r, \alpha} \\
& \times \int_{\pi / 2}^{t}\left(\sin \frac{v}{2}\right)^{-1}\left(\cos \frac{v}{2}\right)^{-(4 s+4 r+1)} \int_{v}^{\pi} \sin \frac{u}{2}\left(\cos \frac{u}{2}\right)^{2 s+2 r+1} d u d v .
\end{aligned}
$$

Поскольку для $\pi / 2 \leqslant t<\pi$ имеем

$$
\int_{\pi / 2}^{t}\left(\sin \frac{v}{2}\right)^{-1}\left(\cos \frac{v}{2}\right)^{-(4 s+4 r+1)} \int_{v}^{\pi} \sin \frac{u}{2}\left(\cos \frac{u}{2}\right)^{2 s+2 r+1} d u d v \leqslant \frac{C_{8}}{\left(\cos \frac{t}{2}\right)^{2 s+2 r}}
$$

то, учитьвая это неравенство, получаем, что

$$
I_{2} \leqslant \frac{C_{9}}{\left(\cos \frac{t}{2}\right)^{2 s+2 r}}\left\|D_{x, s+2 r, s} f(x)\right\|_{p, \alpha+r, \alpha} \leqslant \frac{C_{9} t^{2}}{\left(\cos \frac{t}{2}\right)^{2 s+2 r}}\left\|D_{x, s+2 r, s} f(x)\right\|_{p, \alpha+r, \alpha} .
$$


Так как

$\left\|T_{\cos t}(g, x)-g(x)\right\|_{p, \alpha+r, \alpha} \leqslant\left\|T_{\cos t}(g, x)-T_{0}(g, x)\right\|_{p, \alpha+r, \alpha}+\left\|T_{0}(g, x)-g(x)\right\|_{p, \alpha+r, \alpha}$, то, применяя оценку для $I_{2}$ и оценку для $I$ при $t=\pi / 2$, получаем, что для $\pi / 2 \leqslant t<\pi$ справедливо неравенство

$$
\left\|T_{\cos t}(g, x)-g(x)\right\|_{p, \alpha+r, \alpha} \leqslant \frac{C_{10} t^{2}}{\left(\cos \frac{t}{2}\right)^{2 s+2 r}}\left\|D_{x, s+2 r, s} f(x)\right\|_{p, \alpha+r, \alpha} .
$$

Таким образом, неравенство (7) доказано для $t \in(0, \pi)$. Так как $T_{\cos t}(g, x)=$ $T_{\cos (-t)}(g, x)$, можно сказать, что неравенство $(7)$ справедливо для $0<|t|<\pi$.

Пусть $f \in L_{p, \alpha+r, \alpha}$ и $0<|t| \leqslant \delta<\pi$. Тогда для любой функции $g \in A D(p, \alpha+r, \alpha$, $s+2 r, s)$, применяя теорему 1 , имеем

$$
\begin{aligned}
\left\|T_{\cos t}(f, x)-f(x)\right\|_{p, \alpha+r, \alpha} \leqslant & \left\|T_{\cos t}(f-g, x)\right\|_{p, \alpha+r, \alpha} \\
& +\left\|T_{\cos t}(g, x)-g(x)\right\|_{p, \alpha+r, \alpha}+\|g-f\|_{p, \alpha+r, \alpha} .
\end{aligned}
$$

Применяя теперь теорему 2 и неравенство (7), получаем, что

$$
\left\|T_{\cos t}(f, x)-f(x)\right\|_{p, \alpha+r, \alpha} \leqslant \frac{C_{11}}{\left(\cos \frac{t}{2}\right)^{2 s+2 r}}\left(\|f-g\|_{p, \alpha+r, \alpha}+t^{2}\left\|D_{x, s+2 r, s} g(x)\right\|_{p, \alpha+r, \alpha}\right),
$$

где постоянная $C_{11}$ не зависит от $t, f$ и $g$. Следовательно, переходя к точной нижней грани в этом неравенстве при $|t| \leqslant \delta$ и $g \in A D(p, \alpha+r, \alpha, s+2 r, s)$, получаем справедливость правого неравенства теоремы 3 для $0<\delta<\pi$.

Для доказательства левого неравенства рассмотрим функцию

$$
\begin{aligned}
g_{\delta}(x)= & \frac{1}{K(\delta)} \int_{0}^{\delta}\left(\sin \frac{v}{2}\right)^{-1}\left(\cos \frac{v}{2}\right)^{-(4 s+4 r+1)} \\
& \times \int_{0}^{v} T_{\cos u}(f, x) \sin \frac{u}{2}\left(\cos \frac{u}{2}\right)^{4 s+4 r+1} d u d v
\end{aligned}
$$

где

$$
K(\delta)=\int_{0}^{\delta}\left(\sin \frac{v}{2}\right)^{-1}\left(\cos \frac{v}{2}\right)^{-(4 s+4 r+1)} \int_{0}^{v} \sin \frac{u}{2}\left(\cos \frac{u}{2}\right)^{4 s+4 r+1} d u d v
$$

Применяя обобщенное неравенство Минковского, а затем теорему 2, получаем, что

$$
\left\|g_{\delta}\right\|_{p, \alpha+r, \alpha} \leqslant \frac{C_{12}}{\left(\cos \frac{\delta}{2}\right)^{2 s+2 r}}\|f\|_{p, \alpha+r, \alpha}
$$

т.е. получаем, что $g_{\delta} \in L_{p, \alpha+r, \alpha}$.

Пусть $0<\delta \leqslant \pi / 2$. Тогда легко показать, что

$$
K(\delta) \geqslant C_{13} \delta^{2},
$$

где постоянная $C_{13}$ не зависит от $\delta$. 
В условиях теоремы 3 из того, что $f \in L_{p, \alpha+r, \alpha}$, следует, что $f \in L_{1, s+2 r, s}$. Рассмотрим функцию

$$
g(x)=-\int_{0}^{x}(1-y)^{-(s+2 r+1)}(1+y)^{-(s+1)} \int_{y}^{1}(1-z)^{s+2 r}(1+z)^{s}\left(f(z)-\frac{C_{1}}{C_{0}}\right) d z d y,
$$

где

$$
C_{1}=\int_{-1}^{1} f(z)(1-z)^{s+2 r}(1+z)^{s} d z, \quad C_{0}=\int_{-1}^{1}(1-z)^{s+2 r}(1+z)^{s} d z .
$$

Поскольку $D_{x, s+2 r, s} g(x)=f(x)-C_{1} / C_{0}$, то

$$
\begin{aligned}
g_{\delta}(x)= & \frac{1}{K(\delta)} \int_{0}^{\delta}\left(\sin \frac{v}{2}\right)^{-1}\left(\cos \frac{v}{2}\right)^{-(4 s+4 r+1)} \\
& \times \int_{0}^{v} T_{\cos u}\left(D_{x, s+2 r, s} g(x), x\right) \sin \frac{u}{2}\left(\cos \frac{u}{2}\right)^{4 s+4 r+1} d u d v+\frac{C_{1}}{C_{0}} .
\end{aligned}
$$

Применяя следствие из леммы 1, получаем, что

$$
g_{\delta}(x)=\frac{1}{K(\delta)}\left(T_{\cos \delta}(g, x)-g(x)\right)+\frac{C_{1}}{C_{0}} .
$$

Применяя к этому равенству оператор $D_{x, s+2 r, s}$ и лемму 1 , находим, что

$$
\begin{aligned}
D_{x, s+2 r, s} g_{\delta}(x) & =\frac{1}{K(\delta)}\left(T_{\cos \delta}\left(D_{x, s+2 r, s} g(x), x\right)-D_{x, s+2 r, s} g(x)\right) \\
& =\frac{1}{K(\delta)}\left(T_{\cos \delta}(f, x)-f(x)\right) .
\end{aligned}
$$

Применяя теперь теорему 2 и лемму 1 , заключаем, что $g_{\delta} \in A D(p, \alpha+r, \alpha, s+2 r, s)$.

Из последнего равенства и неравенства (8) получаем, что

$$
\left\|D_{x, s+2 r, s} g_{\delta}(x)\right\|_{p, \alpha+r, \alpha} \leqslant \frac{C_{14}}{\delta^{2}}\left\|T_{\cos \delta}(f, x)-f(x)\right\|_{p, \alpha+r, \alpha},
$$

откуда следует, что

$$
\left\|D_{x, s+2 r, s} g_{\delta}(x)\right\|_{p, \alpha+r, \alpha} \leqslant \frac{C_{14}}{\delta^{2}} \widetilde{\omega}(f, \delta)_{p, \alpha+r, \alpha} .
$$

Теперь оценим

$$
I_{3}=\left\|f-g_{\delta}\right\|_{p, \alpha+r, \alpha} .
$$

Применяя обобщенное неравенство Минковского, имеем

$$
\begin{aligned}
I_{3} \leqslant & \frac{1}{K(\delta)} \int_{0}^{\delta}\left(\sin \frac{v}{2}\right)^{-1}\left(\cos \frac{v}{2}\right)^{-(4 s+4 r+1)} \\
& \times \int_{0}^{v}\left\|f(x)-T_{\cos u}(f, x)\right\|_{p, \alpha+r, \alpha} \sin \frac{u}{2}\left(\cos \frac{u}{2}\right)^{4 s+4 r+1} d u d v \\
\leqslant & \widetilde{\omega}(f, \delta)_{p, \alpha+r, \alpha} .
\end{aligned}
$$


Таким образом, для $0<\delta \leqslant \pi / 2$ показано, что

$$
I(\delta)=\left\|f-g_{\delta}\right\|_{p, \alpha+r, \alpha}+\delta^{2}\left\|D_{x, s+2 r, s} g_{\delta}(x)\right\|_{p, \alpha+r, \alpha} \leqslant C_{15} \widetilde{\omega}(f, \delta)_{p, \alpha+r, \alpha} .
$$

Так как для $\pi / 2 \leqslant \delta<\pi$

$$
\begin{aligned}
K(f, \delta, s+2 r, s) & \leqslant \pi^{2}\left(\left\|f-g_{1}\right\|_{p, \alpha+r, \alpha}+\left\|D_{x, s+2 r, s} g_{1}\right\|_{p, \alpha+r, \alpha}\right)=\pi^{2} I(1) \\
& \leqslant \pi^{2} C_{15} \widetilde{\omega}(f, 1)_{p, \alpha+r, \alpha} \leqslant C_{16} \widetilde{\omega}(f, \delta)_{p, \alpha+r, \alpha},
\end{aligned}
$$

то левое неравенство теоремы 3 справедливо для любого $\delta \in(0, \pi)$.

Теорема 3 доказана полностью.

\section{Оценки модуля гладкости через наилучшие приближения.}

Лемма 2. Пусть даны числа р и $\alpha$ такие, что $1 \leqslant p \leqslant \infty,-1 /(2 p)<\alpha<s+$ $1 / 2-1 /(2 p)$. Пусть $f \in A D(p, \alpha+r, \alpha, s+2 r, s)$. Тогда справедливо неравенство

$$
E_{n}(f)_{p, \alpha+r, \alpha} \leqslant \frac{C}{n^{2}}\left\|D_{x, s+2 r, s} f(x)\right\|_{p, \alpha+r, \alpha},
$$

где постоянная $C$ не зависит от $f$ u $n(n \in \mathbb{N})$.

ДокАЗАТЕльство. Для любого натурального числа $n$ и фиксированного натурального числа $q \geqslant 8$ выберем натуральное число $m$ такое, что

$$
\frac{n-1}{q+2}<m \leqslant \frac{n-1}{q+2}+1
$$

Из условий леммы следует, что $f \in L_{1, s+2 r, s}$. Для этой функции введем симметричньй оператор обобщенного сдвига

$$
\begin{aligned}
\mathscr{T}_{t}(f, x)= & \frac{1}{\gamma} \int_{0}^{1} \int_{-1}^{1} f\left(x \cos t+y z \sin t \sqrt{1-z^{2}}-\left(1-y^{2}\right)(1-x) \sin ^{2} \frac{t}{2}\right) \\
& \times\left(1-y^{2}\right)^{2 r-1} y^{2 s+1}\left(1-z^{2}\right)^{s-\frac{1}{2}} d z d y
\end{aligned}
$$

где $\gamma=\int_{0}^{1} \int_{-1}^{1}\left(1-y^{2}\right)^{2 r-1} y^{2 s+1}\left(1-z^{2}\right)^{s-1 / 2} d z d y$. В работе [6] показано, что функция

$$
Q(x)=\frac{1}{\gamma_{m}} \int_{0}^{\pi} \mathscr{T}_{t}(f, x)\left(\frac{\sin \frac{m t}{2}}{\sin \frac{t}{2}}\right)^{2 q+4}\left(\sin \frac{t}{2}\right)^{2 s+4 r+1}\left(\cos \frac{t}{2}\right)^{2 s+1} d t
$$

где

$$
\gamma_{m}=\int_{0}^{\pi}\left(\frac{\sin \frac{m t}{2}}{\sin \frac{t}{2}}\right)^{2 q+4}\left(\sin \frac{t}{2}\right)^{2 s+4 r+1}\left(\cos \frac{t}{2}\right)^{2 s+1} d t,
$$

есть алгебраический многочлен степени не вьше, чем $n-1$. Поэтому, применяя обобщенное неравенство Минковского, имеем

$$
\begin{aligned}
E_{n}(f)_{p, \alpha+r, \alpha} \leqslant & \|f-Q\|_{p, \alpha+r, \alpha} \\
\leqslant & \frac{1}{\gamma_{m}} \int_{0}^{\pi}\left\|\mathscr{T}_{t}(f, x)-f(x)\right\|_{p, \alpha+r, \alpha} \\
& \times\left(\frac{\sin \frac{m t}{2}}{\sin \frac{t}{2}}\right)^{2 q+4}\left(\sin \frac{t}{2}\right)^{2 s+4 r+1}\left(\cos \frac{t}{2}\right)^{2 s+1} d t .
\end{aligned}
$$


В работе [7] показано, что для всех $t$ справедливо неравенство

$$
\left\|\mathscr{T}_{t}(f, x)-f(x)\right\|_{p, \alpha+r, \alpha} \leqslant C_{1} t^{2}\left\|D_{x, s+2 r, s} f(x)\right\|_{p, \alpha+r, \alpha},
$$

где постоянная $C_{1}$ не зависит от $t$ и $f$. Поэтому

$$
\begin{aligned}
E_{n}(f)_{p, \alpha+r, \alpha} \leqslant & C_{1}\left\|D_{x, s+2 r, s} f(x)\right\|_{p, \alpha+r, \alpha} \\
& \times \frac{1}{\gamma_{m}} \int_{0}^{\pi} t^{2}\left(\frac{\sin \frac{m t}{2}}{\sin \frac{t}{2}}\right)^{2 q+4}\left(\sin \frac{t}{2}\right)^{2 s+4 r+1}\left(\cos \frac{t}{2}\right)^{2 s+1} d t .
\end{aligned}
$$

Учитьвая стандартную оценку ядра Джексона, получаем, что

$$
E_{n}(f)_{p, \alpha+r, \alpha} \leqslant \frac{C_{2}}{m^{2}}\left\|D_{x, s+2 r, s} f(x)\right\|_{p, \alpha+r, \alpha} \leqslant \frac{C_{3}}{n^{2}}\left\|D_{x, s+2 r, s} f(x)\right\|_{p, \alpha+r, \alpha}
$$

где постоянные $C_{2}$ и $C_{3}$ не зависят от $f$ и $n(n \in \mathbb{N})$. Лемма 2 доказана.

Лемма 3. Пусть $\mathscr{P}_{n-1}$ - алгебраический многочлен степени не выше, чем $n-1$. Пусть даны числа $p, \sigma, \alpha$ и $\beta$ такие, что $1 \leqslant p \leqslant \infty, \sigma \geqslant 0$,

$$
\alpha>-\frac{1}{p} \quad u \quad \beta>-\frac{1}{p} \quad \text { npu } 1 \leqslant p<\infty, \quad \alpha \geqslant 0 \quad u \quad \beta \geqslant 0 \quad \text { npu } p=\infty
$$

Тогда справедливы неравенства

$$
\left\|\mathscr{P}_{n-1}^{\prime}\right\|_{p, \alpha+1 / 2, \beta+1 / 2} \leqslant C_{1} n\left\|\mathscr{P}_{n-1}\right\|_{p, \alpha, \beta}
$$

$u$

$$
\left\|\mathscr{P}_{n-1}\right\|_{p, \alpha, \beta} \leqslant C_{2} n^{2 \sigma}\left\|\mathscr{P}_{n-1}\right\|_{p, \alpha+\sigma, \beta+\sigma}
$$

әде постоянные $C_{1}$ и $C_{2}$ не зависят от $n(n \in \mathbb{N})$.

Лемма 3 доказана в работе [13].

Теорема 4. Пусть даны числа $s, r, p$ и а такие, что s и $r$ - натуральные числа, $1 \leqslant p \leqslant \infty, s / 2-1 / 2<\alpha \leqslant s / 2$ npu $p=1, s / 2-1 /(2 p)<\alpha<s / 2+1 / 2-1 /(2 p) n p u$ $1<p<\infty, s / 2 \leqslant \alpha<s / 2+1 / 2$ при $p=\infty$. Пусть функиия $f \in L_{p, \alpha+r, \alpha}$. Тогда для любого натурального п справедливы неравенства

$$
C_{1} E_{n}(f)_{p, \alpha+r, \alpha} \leqslant \widetilde{\omega}\left(f, \frac{1}{n}\right)_{p, \alpha+r, \alpha} \leqslant \frac{C_{2}}{n^{2}} \sum_{\nu=1}^{n} \nu E_{\nu}(f)_{p, \alpha+r, \alpha}
$$

где полохительные постоянные $C_{1}$ и $C_{2}$ не зависят от $f$ u $n$. 
ДокАЗАТЕЛЬСтво. Для любой функщии $g \in A D(p, \alpha+r, \alpha, s+2 r, s)$, применяя лемму 2 , имеем

$$
\begin{aligned}
E_{n}(f)_{p, \alpha+r, \alpha} & \leqslant E_{n}(f-g)_{p, \alpha+r, \alpha}+E_{n}(g)_{p, \alpha+r, \alpha} \\
& \leqslant\|f-g\|_{p, \alpha+r, \alpha}+\frac{C_{3}}{n^{2}}\left\|D_{x, s+2 r, s} g(x)\right\|_{p, \alpha+r, \alpha},
\end{aligned}
$$

где постоянная $C_{3}$ не зависит от $g$ и $n(n \in \mathbb{N})$. Отсюда, переходя к точной нижней грани по всем функциям $g \in A D(p, \alpha+r, \alpha, s+2 r, s)$, а затем применяя теорему 3 , получим, что

$$
E_{n}(f)_{p, \alpha+r, \alpha} \leqslant C_{4} K\left(f, \frac{1}{n}, s+2 r, s\right)_{p, \alpha+r, \alpha} \leqslant C_{5} \widetilde{\omega}\left(f, \frac{1}{n}\right)_{p, \alpha+r, \alpha},
$$

и левое неравенство теоремы 4 доказано.

Докажем теперь правое неравенство теоремы 4 . Пусть $Q_{n}(x)$ - алгебраический многочлен степени не вьше, чем $n-1$, осуществляюший наилучшее приближение функции $f$ в метрике $L_{p, \alpha+r, \alpha}$. Пусть целое число $k$ выбрано так, что

$$
2^{k} \leqslant n<2^{k+1} \text {. }
$$

Поскольку $Q_{2^{k}}(x) \in A D(p, \alpha+r, \alpha, s+2 r, s)$, применяя теорему 3 , получаем, что

$$
\widetilde{\omega}\left(f, \frac{1}{n}\right)_{p, \alpha+r, \alpha} \leqslant C_{6}\left(\left\|f-Q_{2^{k}}\right\|_{p, \alpha+r, \alpha}+\frac{1}{n^{2}}\left\|D_{x, s+2 r, s} Q_{2^{k}}(x)\right\|_{p, \alpha+r, \alpha}\right) .
$$

Так как

$$
D_{x, s+2 r, s} Q_{2^{k}}(x)=\sum_{\nu=0}^{k-1} D_{x, s+2 r, s}\left(Q_{2^{\nu+1}}(x)-Q_{2^{\nu}}(x)\right),
$$

то

$$
\left\|D_{x, s+2 r, s} Q_{2^{k}}(x)\right\|_{p, \alpha+r, \alpha} \leqslant \sum_{\nu=0}^{k-1}\left\|D_{x, s+2 r, s}\left(Q_{2^{\nu+1}}(x)-Q_{2^{\nu}}(x)\right)\right\|_{p, \alpha+r, \alpha} .
$$

Из леммы 3 следует, что

$$
\begin{aligned}
\left\|D_{x, s+2 r, s} Q_{n}(x)\right\|_{p, \alpha+r, \alpha} & \leqslant\left\|\left(1-x^{2}\right) Q_{n}^{\prime \prime}(x)\right\|_{p, \alpha+r, \alpha}+C_{7}\left\|Q_{n}^{\prime}(x)\right\|_{p, \alpha+r, \alpha} \\
& \leqslant C_{8} n^{2}\left\|Q_{n}\right\|_{p, \alpha+r, \alpha} .
\end{aligned}
$$

Поэтому

$$
\begin{aligned}
\left\|D_{x, s+2 r, s} Q_{2^{k}}(x)\right\|_{p, \alpha+r, \alpha} & \leqslant C_{9} \sum_{\nu=0}^{k-1} 2^{2(\nu+1)}\left\|\left(Q_{2^{\nu+1}}(x)-Q_{2^{\nu}}(x)\right)\right\|_{p, \alpha+r, \alpha} \\
& \leqslant 2 C_{9} \sum_{\nu=0}^{k-1} 2^{2(\nu+1)} E_{2^{\nu}}(f)_{p, \alpha+r, \alpha} .
\end{aligned}
$$

Поэтому, учитьвая неравенство (9), имеем

$$
\widetilde{\omega}\left(f, \frac{1}{n}\right)_{p, \alpha+r, \alpha} \leqslant \frac{C_{10}}{n^{2}} \sum_{\nu=0}^{k} 2^{2(\nu+1)} E_{2^{\nu}}(f)_{p, \alpha+r, \alpha} .
$$


Замечая, что для $\nu=1,2, \ldots, k$

$$
\sum_{j=2^{\nu-1}}^{2^{\nu}-1} j E_{j}(f)_{p, \alpha+r, \alpha} \geqslant 2^{2(\nu-1)} E_{2^{\nu}}(f)_{p, \alpha+r, \alpha}
$$

находим, что

$$
\begin{aligned}
\widetilde{\omega}\left(f, \frac{1}{n}\right)_{p, \alpha+r, \alpha} & \leqslant \frac{C_{11}}{n^{2}}\left(E_{1}(f)_{p, \alpha+r, \alpha}+\sum_{\nu=1}^{k} \sum_{j=2^{\nu-1}}^{2^{\nu}-1} j E_{j}(f)_{p, \alpha+r, \alpha}\right) \\
& \leqslant \frac{C_{12}}{n^{2}} \sum_{\nu=1}^{n} \nu E_{\nu}(f)_{p, \alpha+r, \alpha} .
\end{aligned}
$$

Теорема 4 доказана.

\section{СПИСОК ЦИТИРОВАННОЙ ЛИТЕРАТУРЫ}

[1] Ditzian Z., Totik V. Moduli of Smoothness. New York: Springer, 1987.

[2] Löfstrëm J., Peetre J. Approximation theorems connected with generalized translations // Math. Ann. 1969. V. 181. P. 255-268.

[3] Pawelke S. Ein Satz von Jackenschen Typ für algebraische Polynome // Acta Sci. Math. 1972. V. 33. № 3-4. P. 323-336.

[4] Butzer P., Stens R., Wehrens M. Higher order moduli of continuity based on the Jacobi translation operator and best approximation // C. R. Math. Rend. Acad. Sci. Canada. 1980. № 2. P. 83-87.

[5] Потапов М.К. О структурных и конструктивных характеристиках некоторых классов функций // Тр. МИАН. 1974. Т. 131. С. 211-231.

[6] Потапов М.К. О структурных характеристиках классов функций с данным порядком наилучшего приближения // Тр. МИАН. 1975. Т. 134. С. 260-277.

[7] Потапов М. К. О приближении алгебраическими многочленами в интегральной метрике с весом Якоби // Вестн. МГУ. Сер. 1. Матем., мех. 1983. № 3. С. 43-52.

[8] Потапов М. К., Федоров В. М. О теоремах Джексона для обобщенного модуля гладкости // Тр. МИАН. 1985. Т. 172. С. 291-298.

[9] Potapov M.K. The operators of generalized translation in the approximation theory // Proceedings of the II Mathematical Conference in Pristina. Pristina, 1997. P. 27-36.

[10] Потапов М. К. О приближении функций, характеризуемых одним несимметричным оператором обобщенного сдвига // Тр. МИАН. 1999. Т. 227. С. 243-259.

[11] Виленкин Н.Я. Специальные функции и теория представления групп. М.: Наука, 1965.

[12] Бейтмен Г., Эрдейи А. Высшие трансцендентные функции. Т. 2. М.: Наука, 1966.

[13] Халилова Б. А. О некоторых оценках для полиномов // Изв. АН АзССР. Сер. физ. -тех. наук. 1974. № 2. С. 46-55.

Московский государственный университет им. М.В. Ломоносова

Поступило

09.11.1999

Исправленный вариант

03.03.2000 